\section{Caractérisation De Quelques Légumes-Feuilles Les Plus Consommés Dans La Ville De Daloa (Centre-Ouest, Côte d'Ivoire)}

\section{Yao N'zué Benjamin,}

UFR Agroforesterie, Université Jean Lorougnon Guédé, Côte d'Ivoire

\section{Kpata-Konan Nazo Edith,}

UFR Agroforesterie, Université Jean Lorougnon Guédé, Côte d’Ivoire

\section{Guetandé Koné Lacina,}

UFR Agroforesterie, Université Jean Lorougnon Guédé, Côte d'Ivoire

\section{Tano Kablan,}

UFR des Sciences et Technologies des Aliments, Université Nangui Abrogoua, Côte d'Ivoire

Submitted: 29 October 2020

Accepted: 03 December 2020

Published: 31 December 2020

Corresponding author:

Yao N’zué Benjamin

DOI: $10.19044 /$ esj.2020.v16n36p257

(c)

Copyright 2020 Yao N’zué B,

Distributed under Creative Commons

BY-NC-ND 4.0 OPEN ACCES

\section{Cite as:}

Yao N'zué B, Kpata-Konan Nazo E, Guetandé Koné L, Tano K. (2020 Caractérisation De Quelques Légumes-Feuilles Les Plus Consommés Dans La Ville De Daloa (Centre-Ouest, Côte d'Ivoire). European Scientific Journal, ESJ, 16 (36), 1.

https://doi.org/10.19044/esj.2020.v16n36p257

\section{Résumé}

L'objectif de cette étude a consisté de recenser à travers une enquête, les différentes espèces de légumes-feuilles les plus consommés dans la ville de Daloa. Ensuite, déterminer quelques paramètres physico-chimiques, nutritionnels, antinutritionnels et antioxydants de ces légumes-feuilles. L'enquête menée auprès de la population a permis d'identifier quatre légumes-feuilles (,Ipomoea batatas L., Colocasia esculenta L., Spinacia oleracea L.et Manihot esculenta Crantz) qui ont été les plus consommés. Dix-neuf (19) paramètres regroupés en quatre (04) grands groupes [physico-chimiques $(\mathrm{pH}$, acidité, humidité et degré Brix), composés biochimiques (fibres, lipides, protéines, glucides totaux, valeur énergétique, fer et calcium), composés antioxydants (vitamine $\mathrm{C}$, flavonoïde et polyphénols totaux) et composés antinutritionnels (oxalates, phytates, Oxalates/Calcium et Phytates/Fer)] ont fait l'objet d'analyse dans ces légumes-feuilles. Les analyses effectuées indiquent des valeurs de $\mathrm{pH}$ $(6,22 \pm 0,02$ à $6,67 \pm 0,01)$; de taux d'humidité $(78,02 \pm 0,25$ à 93,75 $\pm 0,12$ $\%)$ et de cendre $(0,79 \pm 0,01$ à $0,95 \pm 0,01$ $\%)$. Les minéraux sont importants avec des teneurs en Fer $(15,07 \pm 0,24$ à 40,06 \pm $0,07 \mathrm{mg} / 100 \mathrm{~g})$ et en Calcium $(111,02 \pm$ $0,29$ à $246,28 \pm 0,67 \mathrm{mg} / 100 \mathrm{~g})$. Les analyses biochimiques indiquent de fortes teneurs en glucides $(71,26 \pm 0,25$ à 90,85 $\pm 0,12 \%)$, en protéines ( $1,82 \pm 0,12$ à 5,22 $\pm 0,18 \%)$ et en fibres $(0,55 \pm 0,07$ à 10,00 $\pm 0,14 \%$ ), mais de faibles teneurs en lipides $(0,29 \pm 0,02$ à $0,69 \pm 0,04 \%)$. L'analyse des antioxydants a révélé que la teneur en polyphénol $(107,28 \pm 0,74$ à 195,34 \pm 0,96 mg/100g) est supérieure à 
European Scientific Journal, ESJ

December 2020 edition Vol.16, No.36

celle de la vitamine C $(22,92 \pm 0,72$ à $40,83 \pm 0,72 \mathrm{mg} / 100 \mathrm{~g}$ ) et à celle des flavonoïdes $(2,38 \pm 0,32$ à $7,73 \pm 0,20$ $\mathrm{mg} / 100 \mathrm{~g})$ quel que soit le légume étudié. L'analyse des composés antinutritionnels a révélé une teneur élevée en oxalates $(44,20 \pm 1,27$ à $64,75 \pm 0,52 \mathrm{mg} / 100 \mathrm{~g})$ et en phytates $(48,65 \pm 0,87$ à $65,75 \pm 0,65$ $\mathrm{mg} / 100 \mathrm{~g}$ ) dans les quatre légumesfeuilles. En somme, ces légume-feuilles pourraient constituer un complément alimentaire important, car ils sont une bonne source de minéraux, de fibres, de glucides, de protéine et de composés antioxydants.

\section{Subject: Agriculture}

Mots-clés: Légumes-Feuilles, Enquête, Analyses Physico-Chimiques, Composés Nutritionnels, Composés Antinutritionnels, Composés Antioxydants, Côte d'Ivoire 


\section{Characterization Of Some Of The Most Consumed Leafy Vegetables In The Town Of Daloa (Center- West, Côte d'Ivoire)}

\section{Yao N'zué Benjamin,}

UFR Agroforesterie, Université Jean Lorougnon Guédé, Côte d’Ivoire

Kpata-Konan Nazo Edith,

UFR Agroforesterie, Université Jean Lorougnon Guédé, Côte d’Ivoire

\section{Guetandé Koné Lacina,}

UFR Agroforesterie, Université Jean Lorougnon Guédé, Côte d’Ivoire

\section{Tano Kablan,}

UFR des Sciences et Technologies des Aliments, Université Nangui Abrogoua, Côte d'Ivoire

\section{Abstract}

The objective of this study was to identify through a survey, the different species of leafy vegetables most consumed in the city of Daloa. Then, to determine some physico-chemical, nutritional, antinutritional and antioxidant parameters of these leafy vegetables. The survey conducted among the population identified four leafy vegetables (,Ipomoea batatas L., Colocasia esculenta L., Spinacia oleracea L.and Manihot esculenta Crantz) that were the most consumed. Nineteen (19) parameters grouped into four (04) major groups [physico-chemical ( $\mathrm{pH}$, acidity, moisture and Brix degree), biochemical compounds (fiber, lipids, proteins, total carbohydrates, energy value, iron and calcium), antioxidant compounds (vitamin $\mathrm{C}$, flavonoid and total polyphenols) and anti-nutritional compounds (oxalates, phytates, Oxalates/Calcium and Phytates/Iron)] were analyzed in these leafy vegetables. The analyses performed indicate values for $\mathrm{pH}(6.22 \pm 0.02$ to $6.67 \pm 0.01)$; moisture content (78.02 \pm 0.25 to $93.75 \pm 0.12 \%)$ and ash $(0.79 \pm 0.01$ to 0.95 $\pm 0.01 \%$ ). Minerals are important with Iron (15.07 \pm 0.24 to $40.06 \pm 0.07$ $\mathrm{mg} / 100 \mathrm{~g})$ and Calcium (111.02 \pm 0.29 to $246.28 \pm 0.67 \mathrm{mg} / 100 \mathrm{~g})$ contents. Biochemical analyses indicate high levels of carbohydrates (71.26 \pm 0.25 to $90.85 \pm 0.12 \%)$, protein $(1.82 \pm 0.12$ to $5.22 \pm 0.18 \%)$ and fiber $(0.55 \pm 0.07$ to $10.00 \pm 0.14 \%)$, but low levels of lipids $(0.29 \pm 0.02$ to $0.69 \pm 0.04 \%)$. Antioxidant analysis revealed that polyphenol (107.28 \pm 0.74 to $195.34 \pm 0.96$ $\mathrm{mg} / 100 \mathrm{~g})$ is higher than vitamin C $(22.92 \pm 0.72$ to $40.83 \pm 0.72 \mathrm{mg} / 100 \mathrm{~g})$ 
and flavonoids ( $2.38 \pm 0.32$ to $7.73 \pm 0.20 \mathrm{mg} / 100 \mathrm{~g})$ in all vegetables. The analysis of anti-nutritional compounds revealed high levels of oxalates (44.20 \pm 1.27 to $64.75 \pm 0.52 \mathrm{mg} / 100 \mathrm{~g})$ and phytates $(48.65 \pm 0.87$ to $65.75 \pm 0.65$ $\mathrm{mg} / 100 \mathrm{~g})$ in all four leafy vegetables. In summary, these leafy greens could be an important dietary supplement because they are a good source of minerals, fibre, carbohydrates, protein and antioxidant compounds.

Keywords: Leafy Vegetables, Survey, Physico-Chemical Analyzes, Nutritional Compounds, Antinutritional Compounds, Antioxidant Compounds, Côte D’Ivoire

\section{Introduction}

L'Afrique subsaharienne est dotée d'une grande diversité de plantes alimentaires. Parmi celles-ci se trouvent les légume-feuilles (Almekinders, 2000). Ils jouent un grand rôle dans l'agriculture et dans l'alimentation et génèrent des revenus non négligeables aussi bien dans les zones rurales que dans les zones urbaines (Chweya et Eyzaguirre, 1999). Ils sont d'importantes sources de vitamines (A, B et C), d'oligo-éléments, de protéines, de fibres et de glucides (Chweya et Eyzaguirre, 1999) et de ce fait, contribuent à l'amélioration de l'état nutritionnel des populations aussi bien dans les zones rurales que dans les zones urbaines. Certains légumes-feuilles traditionnels comme Hibiscus sabdariffa (L.) et Ocimum gratissimum (L.) possèdent des propriétés médicinales (Mnzava, 1997 et Vodouhe et al., 2012) et sont utilisés pour soigner diverses maladies (paludisme, parasites intestinaux, infection, etc.). D'autres espèces de légumes par contre comme la patate (Ipomoea batatas L.), l'épinard (Spinacia oleracea), le manioc (Manihot esculenta Crantz) et le taro (Colocasia esculenta L.) sont cultivées pour la consommation (Chweya et Eyzaguirre, 1999). En Côte d’Ivoire, les légumes-feuilles occupent une place de choix dans l'habitude alimentaire. Ils sont cultivés comme plantes maraîchères et vendus sur les marchés. La consommation de ces légumes-feuilles est liée aux différentes régions. Ainsi, sont consommés la corête potagère au Centre ; l'amarante, l'oseille de Guinée, la morelle noire et les feuilles de patate au Nord ; la célosie, l'épinard et le Caya blanc à l'Ouest. Ces légume-feuilles renferment des micronutriments (vitamines, minéraux) qui contribuent au bien-être de l'organisme (Rubaihayo, 1996). Cependant, malgré leurs multiples vertus et le grand intérêt qu'ils suscitent chez certains consommateurs, très peu de données sont disponibles sur leurs compositions physico-chimiques, nutritionnelles, antinutritionnelles et antioxydantes.

C'est dans cette optique que la présente étude a été menée et s'est fixée comme objectif général de caractériser quelques légumes-feuilles les plus consommés dans la ville de Daloa (centre-ouest de la Côte d'Ivoire) afin de 
participer à la sensibilisation des consommateurs et des pouvoirs publics sur les bienfaits de la consommation de ces aliments. De façon spécifique, l'étude a consisté à :

- recenser à travers une enquête, les différentes espèces de légumefeuilles les plus consommés dans la zone d'étude ;

- déterminer quelques paramètres physico-chimiques, nutritionnels et antinutritionnels de ces légumes feuilles.

\section{Matériel et méthodes}

\section{Matériel}

\subsection{Matériel végétal}

Les légumes-feuilles collectés sur les marchés de Gbeul-ville et de lobia (Daloa, Côte d’Ivoire) ont constitué le matériel végétal de cette étude.

\subsection{Site d'étude}

La présente étude s'est déroulée dans le centre-ouest de la Côte d'Ivoire précisément à Daloa, Chef-lieu de la région du Haut-Sassandra (Figure 1). Cette ville a pour coordonnées géographiques $\left(6^{\circ} 27\right.$ de longitudes Ouest et $6^{\circ} 54$ de latitudes Nord). La superficie est de $15200 \mathrm{~km}^{2}$ avec une population de 1.430.960 habitants (BNETD, 2016).

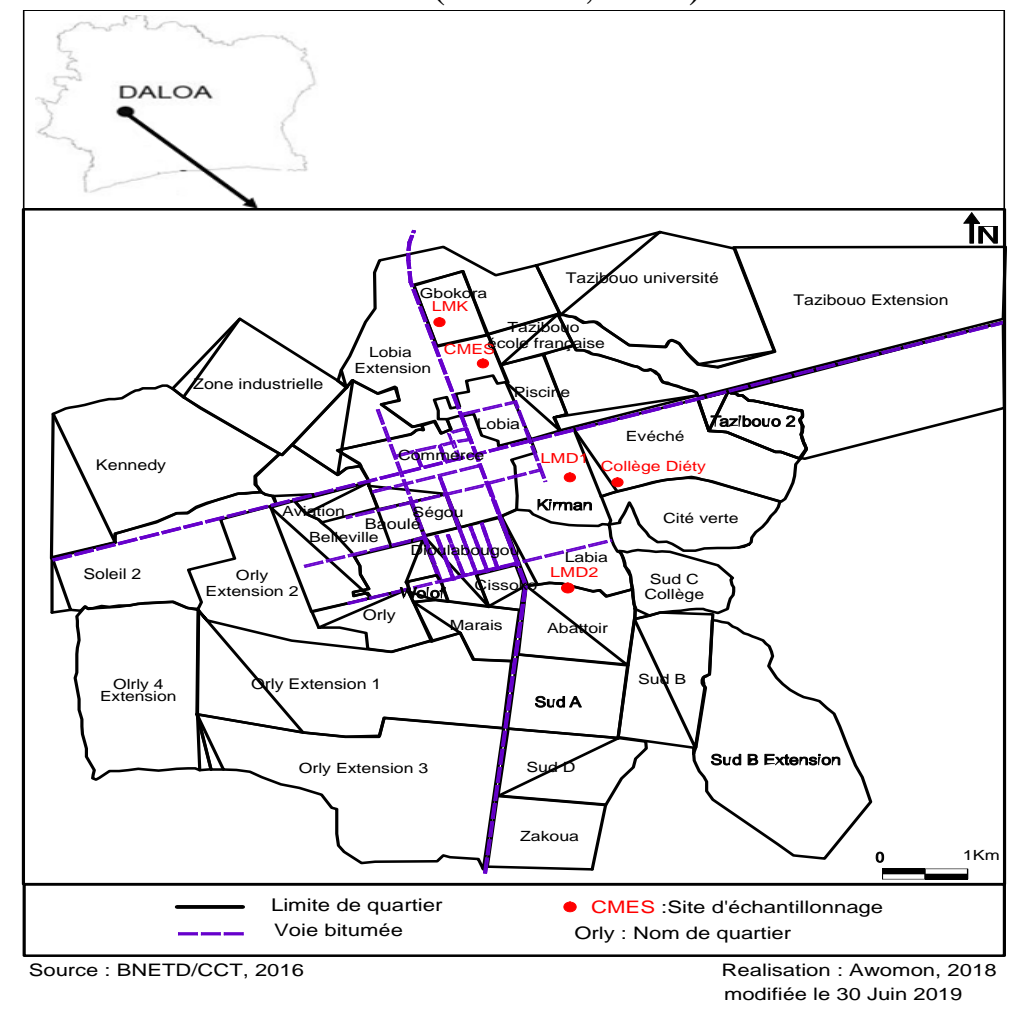

Figure 1. Carte géographique de la ville de Daloa 


\section{Méthodes}

\subsection{Enquête de consommation}

Elle s'est déroulée dans la ville de Daloa. L'enquête a eu pour objectif de recenser les légumes-feuilles les plus consommés par les populations (200 ménages enquêtés) reparties à travers les quartiers de la ville de Daloa (Tazibouo, soleil, Kennedy, Lobia, Belle-ville, Garage, Quartier Baoulé). De plus, le mode de consommation, de préparation et l'intérêt de leur utilisation ont été investigués. La taille des ménages enquêtés a été déterminée à partir de la formule de Vaughan et Morrow (1991) :

$\mathrm{N}=\mathrm{t} 2 \times \mathrm{p}(1-\mathrm{p}) / \mathrm{e} 2$

Avec : $\mathrm{N}=$ taille de l'échantillon attendu $; \mathrm{t}=$ niveau de confiance selon la loi normale centrée réduite $(\mathrm{t}=1,96) ; \mathrm{p}=$ proportion estimée de la population de la ville de Daloa qui consomme les légumes-feuilles ; e = marge d'erreur tolérée (5\%).

\subsection{Collecte des échantillons de légumes-feuilles}

La collecte des échantillons de légumes-feuilles s'est faite au niveau des deux (2) marchés de la ville de Daloa (Côte d'Ivoire). Les légumes-feuilles ont été prélevés tôt le matin et acheminés au laboratoire pour l'analyse des différents paramètres physico-chimiques, biochimiques, antioxydants et antinutritionnels des légumes-feuilles.

\subsection{Préparation des échantillons pour les analyses}

Les feuilles ont été lavées avec de l'eau, pesées à l'aide d'une balance électronique (Sartorius ${ }^{\mathrm{TM}}$ Entris4202I-1S, Fisher Scientific, France), puis broyées à l'aide d'un broyeur (Moulinex Mixeur Blender). Le broyat obtenu a été utilisé pour les analyses.

\subsection{Détermination des Paramètres physico-chimiques}

\subsubsection{Humidité et matière sèche}

La détermination de l'humidité ou de la matière sèche a été réalisée par séchage à l'étuve (AOAC, 1990). Cette méthode consiste à évaporer l'eau contenue dans la matière première par séchage dans une étuve à $105{ }^{\circ} \mathrm{C}$ pendant 24 heures. Les creusets propres, sont séchés à l'étuve puis refroidis au dessiccateur. La masse du creuset vide est alors mesurée. Cinq (5) g de légumes-feuilles contenus dans le creuset sont alors placés dans une étuve à $105 \pm 2{ }^{\circ} \mathrm{C}$ pendant 24 heures. L'ensemble creuset + échantillon séché est refroidi au dessiccateur pendant $30 \mathrm{~min}$. Puis la masse du creuset contenant l'échantillon séché de légumes-feuilles M2 est déterminée. Le pourcentage de matière sèche est calculé selon la formule suivante :

$$
\% \text { Humidité }=\frac{M 1-M 2}{M \rho} \times 100
$$

$$
\% \text { MS= } 100-\% \text { Humidité }
$$

Avec : Me : Masse (g) de l'échantillon ; M1: Masse (g) de l'ensemble (creuset + légumesfeuille) avant étuvage ; M2: Masse (g) de l'ensemble (creuset + légumes-feuille) après étuvage ; MS : Matière sèche. 


\subsubsection{Cendres}

Le taux de cendre a été déterminé par la méthode AOAC (1990). Les matières minérales ou cendres sont les résidus obtenus après la destruction de la substance organique par calcination. Un creuset en silice contenant $5 \mathrm{~g}$ de légumes-feuilles est introduit dans un four à moufle, préalablement chauffé à $550 \pm 15^{\circ} \mathrm{C}$ pendant 12 heures. Les cendres blanches obtenues sont refroidies avec le creuset dans un dessiccateur à la température ambiante puis le creuset est pesé après le refroidissement. Le taux de cendres est donné par la formule suivante :

$$
\text { Cendres }(\%)=\frac{(M 2-M 0) \times 100}{M 1-M 0}
$$

Avec : M0 : Masse (g) du creuset vide ; M1 : Masse (g) de l'ensemble (creuset + échantillon) avant incinération ; M2 : Masse (g) de l'ensemble (creuset + cendres) après incinération

\subsubsection{Minéraux (Calcium et Fer)}

\subsubsection{Teneur en Calcium}

Le dosage du calcium est effectué par la méthode titrimétrique à l'EDTA selon la Norme Française d'Août 1984 (AFNOR, 1984). Le titrage des ions calcium est effectué avec une solution aqueuse de sel disodique d'acide éthylène-diamine tétra acétique (EDTA) à un $\mathrm{pH}$ compris entre 12 et 13. Le HSN (Helper Search Token), qui forme un complexe rouge avec le calcium, est utilisé comme indicateur. Le calcium est précipité sous forme d'hydroxyde et n'interfère pas lors du dosage. Lors du titrage, l'EDTA réagit tout d'abord avec les ions calcium libres, puis avec les ions calcium combinés avec l'indicateur qui vire alors de la couleur rouge à la couleur bleu clair. A l'aide d'une pipette, une quantité de $50 \mathrm{~mL}$ de solution de cendre est introduit dans une fiole conique de $250 \mathrm{~mL}$. Après ajout de $2 \mathrm{~mL}$ de la solution d'hydroxyde de sodium ( $2 \mathrm{~N}$ ), environ $0,2 \mathrm{~g}$ de l'indicateur HSN (l'acide calcone carboxylique + chlorure de sodium $(\mathrm{NaCl})$ ) est ajouté. La solution est homogénéisée et le titrage a lieu immédiatement. La solution d’EDTA est ajoutée, tout en continuant l'agitation. Le virage est atteint lorsque la couleur devient nettement bleue. La couleur ne doit plus changer avec l'ajout d'une goutte supplémentaire de la solution d'EDTA. La teneur en Calcium de l'échantillon est donnée en $\mathrm{mg} / 100 \mathrm{~g}$ par l'expression suivante :

Calcium $(\mathrm{mg} / 100 \mathrm{~g})=\left(\mathrm{C} \times \mathrm{V} \_1 \times \mathrm{V}\right) /\left(\mathrm{V} \_0 \times \mathrm{M} \times \mathrm{m} \_\mathrm{e} \times \mathrm{MS}\right) \times 100$

$$
\text { Calcium }(\mathbf{m g} / \mathbf{1 0 0} \mathrm{g})=\frac{\mathrm{C} \times \mathrm{V}_{\mathbf{1}} \times \mathrm{V}}{\mathrm{V}_{\mathbf{0}} \times \mathrm{M} \times \mathrm{m}_{\mathrm{e}} \times \mathrm{MS}} \times \mathbf{1 0 0}
$$

Avec: C : Concentration (mmol/L) de la solution d'EDTA ; Vo : Volume $(\mathrm{mL})$ de la prise d'essai; V1 : Volume (mL) de la solution d’EDTA utilisé pour le dosage ; M : Masse 
atomique relative du calcium $(40,08) ; \mathrm{V}$ : Volume $(\mathrm{mL})$ de l'échantillon ; me : Masse (g) de l'échantillon et MS: Matière sèche.

\subsubsection{Teneur en Fer}

L'analyse du Fer est effectuée grâce au spectrophotomètre de marque HACH DR 3900 selon la méthode TPTZ (2, 4, 6-Tripyridyl-S-triazine). Deux (2) cuves carrées de 1 pouce sont préparées. L’une des deux cuves est la cuve réservée au blanc et l'autre cuve réservée à l'échantillon. Un volume de $10 \mathrm{~mL}$ d'eau déminéralisée est versé dans la cuve du blanc et $10 \mathrm{~mL}$ d'échantillon sont versés dans la cuve réservée à l'échantillon. Dans chaque cuve, une pochette de réactif pour le fer au TPTZ est versée et le contenu est mélangé pendant 30 secondes tout en agitant. Un temps de réaction de 3 minutes est programmé. Après l'écoulement de ce temps, la cuve du blanc est placée dans la chambre de mesure pendant quelque seconde et est retirée. La cuve réservée à l'échantillon est placée à son tour dans la chambre de mesure pendant quelques secondes et est retirée. Le résultat de la mesure s'affiche à l'écran du spectrophotomètre.

\subsubsection{Potentiel d'hydrogène}

La méthode AOAC (1990) a été utilisée dans cette détermination. Le $\mathrm{pH}$ de chaque d'échantillon de légumes-feuilles a été mesuré avec un $\mathrm{pH}$ mètre numérique. Dix (10) g d'échantillon de légumes sont broyés dans 100 $\mathrm{mL}$ d'eau distillée. Le broyat est centrifugé à 3000 tours/min pendant $30 \mathrm{~min}$. Le surnageant est recueilli dans un bocal, le $\mathrm{pH}$ est lu sur un écran digital en plongeant directement l'électrode du $\mathrm{pH}$-mètre dans la solution après avoir étalonné le pH-mètre.

\subsubsection{Acidité titrable}

L’acidité titrable a été déterminée selon la méthode décrite par la norme française NF v05-101 (AFNOR, 1974). Cette mesure est réalisée par neutralisation de l'acidité libre totale avec une solution d'hydroxyde de sodium $(\mathrm{NaOH} ; 0,1 \mathrm{~N})$. L'évolution de la neutralisation est suivie à l'aide d'un indicateur coloré (phénolphtaléine). Le dosage est arrêté lorsque l'indicateur vire au rose/orangé. Cinq (5) g de l'échantillon sont broyés dans $50 \mathrm{~mL}$ d'eau distillée. Le broyat est centrifugé à 4000 tours/min pendant 20 min. Dix (10) mL de surnageant est recueilli dans un erlenmeyer puis 2 gouttes de phénolphtaléine sont versées dans la solution. La soude y est versée goutte à goutte jusqu'au virage rose/orangé. Le résultat est exprimé par l'équation suivante :

$$
\text { Acidité }(\mathrm{meq} / \mathrm{100g})=\frac{N \times V_{1} \times 10^{5}}{m \times V_{0}}
$$

Avec : V1: Volume de $\mathrm{NaOH}$ versé à l'équivalence ; V0: Volume de la prise d'éssai ; m: masse de feuilles fraîches ; $\mathrm{N}$ : Normalité de $\mathrm{NaOH}(0,1 \mathrm{~N})$. 


\subsubsection{Degré Brix}

Le degré Brix est défini comme étant la concentration en hydrates de carbone (principalement le saccharose) d'une solution aqueuse ayant le même indice de réfraction que le produit analysé. Pour l'analyse, quelques gouttes d'extraits de légumes-feuilles ont été déposées sur le prisme fixe d'un réfractomètre. La lecture a été effectuée par observation contre une source lumineuse.

\subsection{Dosage des composés biochimiques (\%)}

\subsubsection{Lipides totaux}

Les lipides sont extraits selon la méthode utilisant le SOXHLET (AFNOR, 1986). Les lipides totaux sont extraits par l'hexane (solvant organique) à partir du broyat de l'échantillon. Dix (10) g d'échantillon broyé ont été introduits dans une cartouche d'extraction préalablement tarée puis bouché par du coton et le tout placé dans l'extracteur de type Soxhlet. L'extraction des lipides totaux a été faite par $300 \mathrm{~mL}$ d'hexane pendant 7 heures à ébullition. Après, l'hexane a été évaporé à l'aide d'un évaporateur rotatif. Ensuite, le ballon d'extraction préalablement taré a été séché à l'étuve à $100{ }^{\circ} \mathrm{C}$ pendant 20 min et l'ensemble (huile - ballon) a été pesé. Enfin, la teneur en lipides a été déterminée par la formule mathématique suivante :

$$
\operatorname{Lipides}(\%)=\frac{\left(m 1-m_{0}\right) \times 100}{m_{e}}
$$

Avec : m0 : Masse (g) du ballon vide ; me : Masse (g) de l'échantillon ; m1 : Masse (g) de l'ensemble (ballon + lipides) après évaporation.

\subsubsection{Protéines totales}

La teneur en protéines brutes est déterminée à partir de la teneur en azote selon la méthode de Kjeldahl (AOAC, 1990). Elle comprend une phase de minéralisation, suivie d'une phase de distillation et d'une phase de titrage par l'acide sulfurique en présence d'indicateur coloré. D’abord une masse de $1 \mathrm{~g}$ de légume est pesée dans un matras de minéralisation auquel sont ajoutés une pincée des catalyseurs (sélénium, sulfate de cuivre (CuSO4) et de sulfate de potassium (K2SO4) et $20 \mathrm{~mL}$ d'acide sulfurique concentré). La minéralisation est effectuée à $400{ }^{\circ} \mathrm{C}$ pendant 2 heures dans un digesteur. Le minéralisât obtenu a été refroidi à la température ambiante et transvasé dans une fiole de $100 \mathrm{~mL}$ et complété avec l'eau distillée. Après avoir mis le distillateur en marche, $10 \mathrm{~mL}$ du minéralisât sont prélevés, auxquels sont ajoutés $10 \mathrm{~mL}$ de $\mathrm{NaOH}$ (40\%). Le mélange est mis dans le réservoir du distillateur. L'allonge du réfrigérant est plongée dans un bécher contenant 20 mL d'acide borique (4\%) additionnée d'indicateur mixte (rouge de méthyle + vert de bromocrésol) : la solution devient violette. La distillation est effectuée 
pendant 10 minutes. A la fin de la distillation, la solution devient verte. Le taux de protéines est déterminé selon la formule suivante :

Avec : V0 : Volume $(\mathrm{mL})$ de solution d'acide sulfurique versé pour l'essai à blanc ; V1 :

$$
\text { Protéines totales }(\%)=\frac{(\mathrm{V} 1-\mathrm{v0}) \times 14 \times 6,25 \times \mathrm{N}}{\mathrm{me}} \times 100
$$

Volume (mL) de solution d'acide sulfurique versé pour l'essai (échantillon) ; N : Normalité de la solution d'acide sulfurique: 0,1; me : Masse (g) de l'échantillon ; 6,25 : Facteur de conversion de l'azote en protéines (FAO, 1978).

\subsubsection{Glucides}

Les glucides totaux en pourcentage de masse d'échantillon frais sont obtenus par la relation suivante :

Glucides (\%) = 100 - [protéines (\%) + lipides (\%) + humidité (\%) + cendres

\subsubsection{Fibres brutes}

La teneur en fibres est déterminée selon la méthode AOAC (1990). Deux (2) grammes de l'échantillon séché et broyé ont été homogénéisé dans $50 \mathrm{~mL}$ d'acide sulfurique $0,25 \mathrm{~N}$ et porté à ébullition pendant 30 minutes et après $50 \mathrm{~mL}$ de soude $0,31 \mathrm{~N}$ ont été ajoutés au mélange précédant en ébullition pendant encore $30 \mathrm{~min}$. Ensuite l'extrait a été filtré à chaud sur un entonnoir muni de papier-filtre préalablement taré et sans résidu. Le résidu a été lavé trois fois avec de l'eau distillée chaude, puis séché à l'étuve à $105^{\circ} \mathrm{C}$ pendant 8 heures. Le papier-filtre a été refroidi au dessiccateur et pesé. Il a été calciné à $550^{\circ} \mathrm{C}$ pendant 3 heures au four à moufle. La cendre a été pesée. La teneur en fibres est donnée par la formule suivante :

$$
\text { Fibres }(\%)=\frac{(m 1-m 0)}{m e} \times 100
$$

Avec : m1 : Masse (g) du résidu séché ; m0 : Masse (g) des cendres obtenues ; me : Masse (g) de l'échantillon.

\subsubsection{Valeur énergétique}

La valeur énergétique a été calculée par application des coefficients thermiques d'Atwater \& Rosa (1899) :

- $1 \mathrm{~g}$ de glucide fournit $4 \mathrm{Kcal}$;

- $1 \mathrm{~g}$ de protéine fournit $4 \mathrm{Kcal}$;

- $1 \mathrm{~g}$ de lipide fournit $9 \mathrm{Kcal}$. 
A partir de ces données, la somme des valeurs énergétiques des éléments nutritifs est calculée pour obtenir la valeur énergétique de l'aliment considéré. Ainsi, pour $100 \mathrm{~g}$ d'aliment, la valeur énergétique a été exprimée comme suit:

\section{$\mathrm{E}$ (Calories) $=4 \times \%$ Protéine $+4 \times \%$ Glucides totaux $+9 \times \%$ Lipide}

\subsection{Dosage des composés antioxydants}

\subsubsection{Vitamine $\mathrm{C}$}

La méthode utilisée pour le dosage de la vitamine $\mathrm{C}$ de nos échantillons est celle décrite par pongracz et al. (1971) dont le principe est basé sur la réduction du 2,6 DCPIP (2,6 dichlorophénol-indophénol) par celle-ci. Dix (10) grammes d'échantillons sont pesés et broyés puis solubilisés dans $40 \mathrm{~mL}$ d'acide métaphosphorique-acide acétique ( $2 \%$; p/v). Le mélange obtenu est centrifugé à $3000 \mathrm{trs} / \mathrm{min}$ pendant $20 \mathrm{~min}$. Le surnageant est introduit dans une fiole jaugée de $50 \mathrm{~mL}$ et est ajusté avec de l'eau distillée bouillie et refroidie à l'abri de l'air. Une prise d'essai de $10 \mathrm{~mL}$ est introduite dans un erlenmeyer puis titrée avec le 2,6 DCPIP à 0,5 g/L jusqu'au virage au rose persistant. La solution de 2,6 DCPIP est préalablement étalonnée avec une solution de vitamine $C$ pure à $0,5 \mathrm{~g} / \mathrm{L}$. La teneur en vitamine $\mathrm{C}$ de l'échantillon est donnée en pourcentage par l'expression suivante :

$$
\text { Vitamine } \boldsymbol{C}(\%)=\frac{\left(0,5 \times V \times 10^{-3}\right) \times 5 \times 100}{m_{e}}
$$

Avec : V : Volume (mL) de 2,6 DCPIP versé à l'équivalence ; me : masse (g) de l'échantillon de feuilles fraîches.

\subsubsection{Phénols totaux}

La méthode de Singleton et al. (1999), utilisant le Folin-ciocalteu, a permis de doser les phénols totaux. Le réactif est constitué par un mélange d'acide phosphotungstique (H3PW12O40) et d'acide phosphomolybdique (H3PMo12O40). Il est réduit, lors de l'oxydation des phénols, en un mélange d'oxydes bleus de tungstène et de molybdène. La coloration produite est proportionnelle à la quantité de polyphénols présents dans les extraits végétaux. Un (1) mL d'extrait méthanolique est introduit dans un tube à essai. Au contenu du tube, est ajouté $1 \mathrm{~mL}$ de réactif de Folin-ciocalteu. Le tube est laissé au repos pendant $3 \mathrm{~min}$ puis $1 \mathrm{~mL}$ de solution de carbonate de sodium à $20 \%$ (p/v) y est ajouté. Le contenu du tube est complété à $10 \mathrm{~mL}$ avec de l'eau distillée. Le tube est placé à l'obscurité pendant 30 min et la lecture de la DO est effectuée à $725 \mathrm{~nm}$ contre un blanc. Une gamme étalon établie à partir d'une solution mère d'acide gallique $(1 \mathrm{mg} / \mathrm{mL})$ dans les mêmes conditions que l'essai a permis de déterminer la quantité de phénols de l'échantillon. 


\subsubsection{Flavonoïdes}

Le dosage des flavonoïdes a été effectué suivant la méthode décrite par Meda et al. (2005). Selon le principe, les flavonoïdes réagissent avec le chlorure d'aluminium en présence d'acétate de potassium pour donner un complexe de couleur jaune dont l'intensité est proportionnelle à la quantité de flavonoïdes présente dans le milieu. Un volume de $0,5 \mathrm{~mL}$ d'extrait méthanolique est introduit dans un tube à essai. Au contenu du tube sont ajoutés successivement $0,5 \mathrm{~mL}$ d'eau distillée, $0,5 \mathrm{~mL}$ de chlorure d'aluminium à $10 \%$ (p/v), 0,5 mL d'acétate de potassium à $1 \mathrm{M}$ et $2 \mathrm{~mL}$ d'eau distillée. Le tube est laissé au repos pendant 30 min à l'obscurité et la densité optique (DO) est lue à $415 \mathrm{~nm}$ contre un blanc. Une gamme étalon établie à partir d'une solution mère de quercetine $(0,1 \mathrm{mg} / \mathrm{mL})$ dans les mêmes conditions que l'essai a permis de déterminer la quantité de flavonoïdes de l'échantillon.

\subsection{Dosage des composés antinutritionnels}

\subsubsection{Phytates}

Le dosage des phytates a été effectué selon la méthode de Latta \& Eskin (1980) utilisant le réactif de Wade. Pour ce faire, un (1) g d'échantillon séché et broyé puis homogénéisé dans $20 \mathrm{~mL}$ de $\mathrm{HCl}(0,65 \mathrm{~N})$ sous agitation, pendant $12 \mathrm{~h}$ à température ambiante. Après centrifugation du mélange à 12000 tours/min pendant $40 \mathrm{~min}, 5 \mathrm{~mL}$ du surnageant a été prélevé puis ajouté à $3 \mathrm{~mL}$ de réactif de Wade. Ensuite, laisser au repos pendant $15 \mathrm{~min}$ et lire la DO au spectrophotomètre à $490 \mathrm{~nm}$ contre le témoin. Réaliser enfin, une gamme d'étalonnage avec le phytate de sodium à $10 \mu \mathrm{g} / \mathrm{mL}$

$$
\text { Phytates }(\mathrm{mg} / 100 \mathrm{~g})=\frac{D O_{490} \times 4}{0,033 \times m_{e}}
$$

Avec: me : masse (g) de l'échantillon ; 0,033 : pente de la droite d'étalonnage ; 4 : facteur de dilution.

\subsubsection{Oxalates}

La méthode utilisée pour le dosage de l'oxalate dans les échantillons de légumes-feuilles est celle décrite par Day \& Underwood (1986). Pour ce faire, un (1) g de poudre de légumes-feuilles est homogénéisé dans $15 \mathrm{~mL}$ de H2SO4 (3M) sous agitation magnétique pendant $1 \mathrm{~h}$. Le mélange obtenu est filtré sur papier filtre Whatman. Le filtrat est titré à chaud avec une solution de $\mathrm{KMnO} 4(0,05 \mathrm{M})$ jusqu'au virage au rose persistant. La teneur en oxalates de l'échantillon est donnée en mg/100 g par l'expression suivante: 


$$
\text { Oxalates }(\mathrm{mg} / 100 \mathrm{~g})=\frac{1,1 \times \mathrm{V}_{\mathrm{eq}} \times 100}{\mathrm{~m}_{\mathrm{e}} \times \mathrm{MS}}
$$

Avec : Veq : Volume (mL) de KMnO4 versé à l’équivalence ; me : Masse (g) de l'échantillon ; MS : Matière sèche.

\subsection{Analyse statistique}

Toutes les mesures ont été effectuées en triple et les moyennes des données ont été analysées statistiquement en utilisant le logiciel STATISTICA 7.1. L'analyse de la variance à un facteur (ANOVA) a été effectuée pour comparer les moyennes. Les différences ont été considérées significatives pour les valeurs de $\mathrm{p} \leq 0,05$. Pour séparer les différentes moyennes des échantillons, des tests de comparaison multiples (Tukey HSD) ont été réalisés.

\section{Résultats Et Discussion}

\section{Résultats}

\subsection{Enquête de consommation}

Une diversité de légumes-feuilles a été recensée dans la localité de Daloa (Figure 2). Les plus consommés ont été : les feuilles de patate douce (23\%), les feuilles de taro (21\%), les feuilles de épinard (18\%), les feuilles de manioc (17 \%), les feuilles de kplala (corète potagère) (10 \%), les feuilles de dah (roselle) (7 \%) et les feuilles de moringa (4\%).

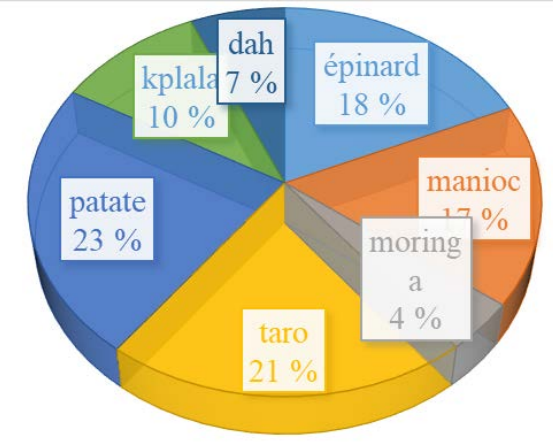

Figure 2: Quelques légumes-feuilles les plus consommés dans la ville de Daloa (CentreOuest, Côte d'Ivoire)

Les résultats des enquêtes ont également montré que 100 \% des ménages connaissent les légumes-feuilles. Ceux-ci sont consommés cuits. De plus, 98 \% des ménages les apprécient comme étant bon pour la santé (Tableau I).

Au niveau du mode de préparation, les résultats ont montré que $80 \%$ des ménages les consomment sous forme de sauce et $20 \%$ en pâte. Pour l’obtention de ces légumes, les résultats ont révélé que 36 \% des ménages 
enquêtés les obtiennent par achat et $64 \%$ des ménages les cultivent. Au niveau de la fréquence de consommation, 53 \% des familles enquêtées les consomment 1 à 2 fois/mois, $36 \%$ les consomment 3 à 4 fois/mois et $11 \%$ les consomment plus de 4 fois/mois. Par ailleurs, pour $70 \%$ des ménages enquêtés ces légumes apportent du sang et pour $30 \%$ des ménages, ils apportent de la vitamine (Tableau I).

Tableau I. Connaissance, consommation, mode de consommation et de préparation, fréquence de consommation, appréciation du légume, obtention du légume, apport du légume et conservation

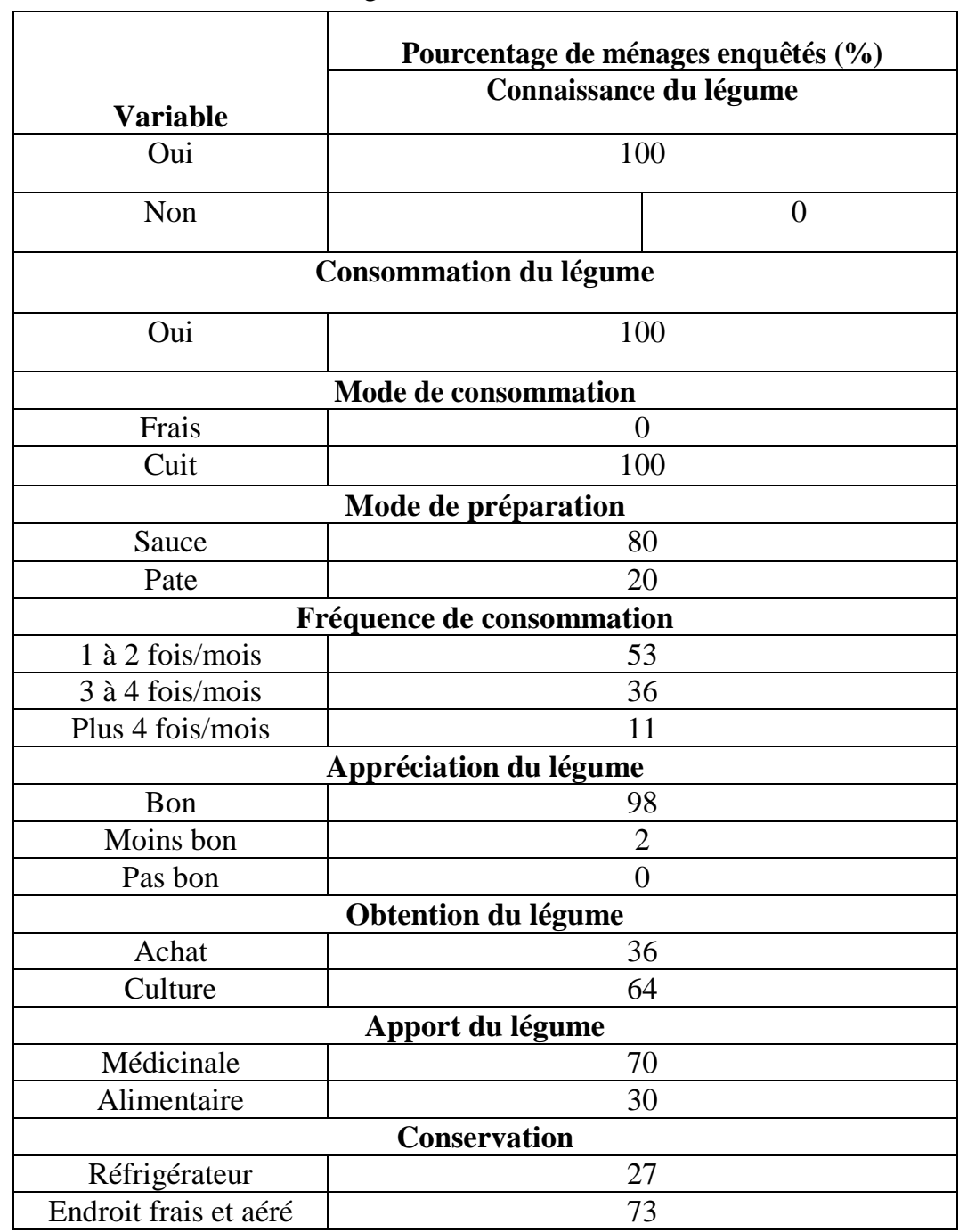


Aussi selon les résultats de l'enquête, les quatre légumes-feuilles les plus consommées sont les feuilles de patate douce, de taro, d'épinard et de manioc (Figure 3).
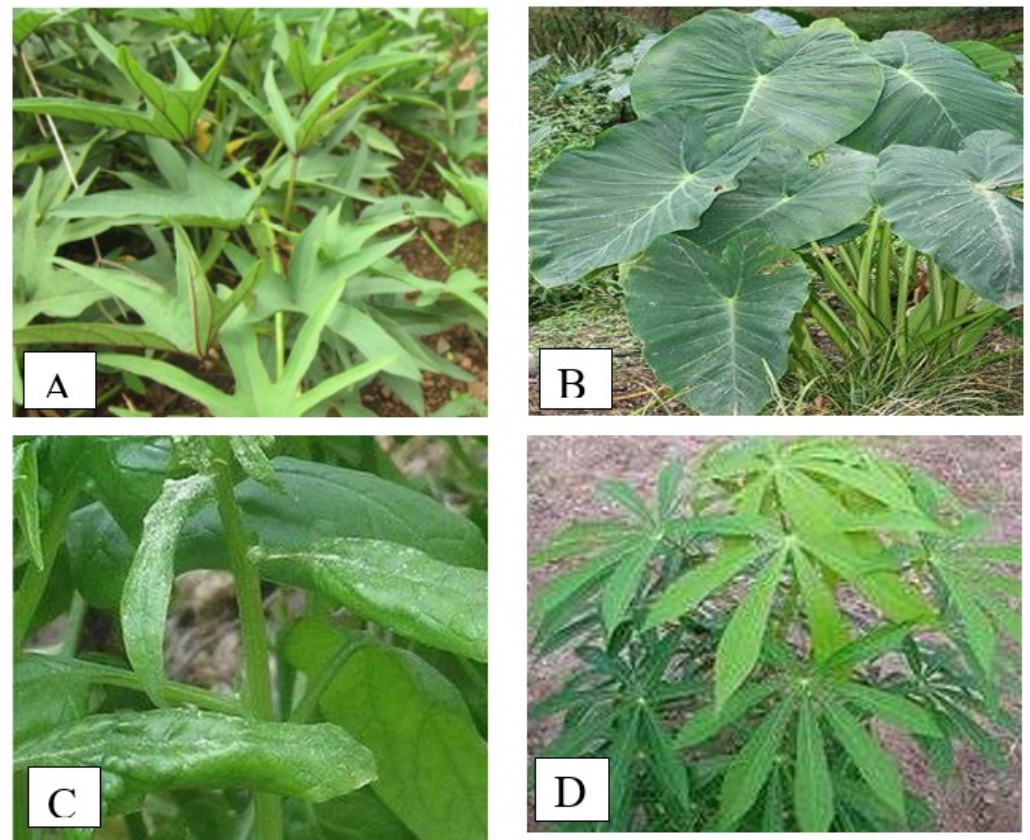

Figure 3. Les quatre légumes-feuilles les plus consommés à Daloa (Centre-Ouest, Côte d'Ivoire) A :Ipomoea batatas L. B : Colocasia esculenta L. C : Spinacia oleracea L. D : Manihot esculenta Crantz.

\subsection{Paramètres physico-chimiques des légumes-feuilles}

L'analyse statistique a montré une différence significative $(p \leq 0,05)$ au niveau du $\mathrm{pH}$. Selon le test HSD de Tukey, les légumes-feuilles de taro, épinard, patate et manioc de $\mathrm{pH}$ respectif $6,67 \pm 0,01 ; 6,22 \pm 0,02 ; 6,56 \pm$ 0,02 et $6,37 \pm 0,01$ sont statistiquement différents (Tableau II). Le taro présente le $\mathrm{pH}$ le plus élevé $(6,67 \pm 0,01)$ et l'épinard, la valeur la plus faible $(6,22 \pm 0,02)$.

L'acidité de ces quatre légumes-feuilles (taro, épinard, patate et manioc) de teneur respective de 46,67 $\pm 11,55 \mathrm{meq} / 100 \mathrm{~g} ; 60,00 \pm 0,00$ meq/100 g ; 46,67 $\pm 11,55 \mathrm{meq} / 100 \mathrm{~g}$ et $53,33 \pm 11,55 \mathrm{meq} / 100 \mathrm{~g}$ est statistiquement identique ( $\mathrm{p} \geq 0,05)$ (Tableau II).

Par ailleurs, l'analyse statistique a montré une différence significative ( $\mathrm{p} \leq$ $0,05)$ au niveau du taux d'humidité. Les légumes-feuilles de taro, épinard, patate et manioc de teneurs respectives $87,88 \pm 0,32 \% ; 93,75 \pm 0,12 \%$; $86,42 \pm 0,26 \%$ et 78, $02 \pm 0,25 \%$ sont statistiquement différents (Tableau II). L'épinard présente le taux d'humidité le plus élevé (93,75 \pm 0,12\%) pendant que le manioc enregistre le taux le plus faible (78,02 $\pm 0,25 \%)$. 
Le taux de cendres a été analysé. L’analyse statistique a montré une différence significative $(\mathrm{p} \leq 0,05)$. Selon le test HSD de Tukey, les légumesfeuilles de taro, épinard, patate et manioc de teneurs respectives $0,91 \pm 0,01$ $\% ; 0,79 \pm 0,01 \% ; 0,89 \pm 0,01 \%$ et $0,95 \pm 0,01 \%$ sont statistiquement différents (Tableau II). Toutefois, les feuilles de taro et patate ont enregistré des teneurs statistiquement identiques. Le manioc présente le taux de cendre le plus élevé $(0,95 \pm 0,01 \%)$ et l'épinard le taux le plus faible $(0,79 \pm 0,01 \%)$. Concernant le degré Brix, l'analyse statistique a montré une différence significative $(\mathrm{p} \leq 0,05)$. Les degrés Brix des feuilles de taro, épinard, patate et manioc de valeur respective $3,23 \pm 0,25{ }^{\circ}$ Brix ; 3,16 $\pm 0,29{ }^{\circ}$ Brix; 2,16 $\pm 0,47$ ${ }^{\circ}$ Brix et 7,00 $\pm 0,00{ }^{\circ}$ Brix sont statistiquement différents. Néanmoins, Les feuilles de taro et épinard ont des valeurs statistiquement identiques.

Tableau II : Paramètres physico-chimiques des légumes-feuilles.

Paramètres physico-chimiques

\begin{tabular}{cccccc}
\hline $\begin{array}{c}\text { Légumes- } \\
\text { feuilles }\end{array}$ & pH & $\begin{array}{c}\text { Acidité } \\
(\mathbf{m e q} / \mathbf{1 0 0} \text { g) }\end{array}$ & $\begin{array}{c}\text { Humidité } \\
\mathbf{( \% )}\end{array}$ & Cendres (\%) & Degré Brix \\
\hline Taro & $6,67 \pm$ & $46,67 \pm$ & $87,88 \pm$ & $0,91 \pm 0,01^{\mathrm{a}}$ & $3,23 \pm 0,25^{\mathrm{a}}$ \\
& $0,01^{\mathrm{a}}$ & $\begin{array}{c}11,55^{\mathrm{a}} \\
0,32^{\mathrm{a}}\end{array}$ & & \\
Epinard & $6,22 \pm$ & $60,00 \pm 0,00^{\mathrm{a}}$ & $\begin{array}{c}93,75 \pm \\
0,12^{\mathrm{b}}\end{array}$ & $0,79 \pm 0,01^{\mathrm{b}}$ & $3,16 \pm 0,29^{\mathrm{a}}$ \\
& $0,02^{\mathrm{b}}$ & & & & \\
Patate & $6,56 \pm$ & $46,67 \pm$ & $86,42 \pm$ & $0,89 \pm 0,01^{\mathrm{a}}$ & $2,16 \pm 0,47^{\mathrm{b}}$ \\
& $0,02^{\mathrm{c}}$ & $11,55^{\mathrm{a}}$ & $0,26^{\mathrm{c}}$ & & \\
Manioc & $6,37 \pm$ & $53,33 \pm$ & $78,02 \pm$ & $0,95 \pm 0,01^{\mathrm{c}}$ & $7,00 \pm 0,00^{\mathrm{c}}$ \\
\hline
\end{tabular}

Les valeurs avec les lettres alphabétiques dans la même colonne sont statistiquement identiques au seuil de $5 \%$ selon le test HSD de Tukey

\subsection{Paramètres biochimiques des légumes-feuilles}

L'analyse statistique a montré une différence significative $(\mathrm{p} \leq 0,05)$ au niveau des teneurs en lipides des échantillons analysés. Selon le test HSD de Tukey, les feuilles de taro, épinard, patate et manioc de teneurs respectives $0,62 \pm 0,04 \% ; 0,29 \pm 0,02 \% ; 0,46 \pm 0,01 \%$ et $0,69 \pm 0,04 \%$ sont statistiquement différents (Tableau III). Toutefois, les légumes-feuilles de patate et manioc de teneurs respectives $0,46 \pm 0,01 \%$ et $0,69 \pm 0,04 \%$ sont statistiquement identiques. Le manioc a présenté la teneur la plus élevée $(0,69$ $\pm 0,04 \%)$ et l'épinard, la teneur la plus faible $(0,29 \pm 0,02 \%)$ (Tableau III). Tout comme les lipides, la teneur en protéines des légumes-feuilles étudiés diffère significativement $(\mathrm{p} \leq 0,05)$. Pendant que le manioc a présenté la valeur la plus élevée (5,22 $\pm 0,18 \%)$, l'épinard a enregistré, la plus faible (1,82 \pm $0,12 \%)$.

Par ailleurs, l'analyse statistique a révélé une différence significative $(\mathrm{p} \leq 0,05)$ au niveau des teneurs en glucides des échantillons analysés. Ainsi, les feuilles de taro, épinard, patate et manioc avec des teneurs respectives 
$82,47 \pm 0,46 \% ; 90,85 \pm 0,12 \% ; 82,45 \pm 0,26 \%$ et $71,26 \pm 0,25 \%$ sont statistiquement différents (Tableau III). De plus, les feuilles de taro et de patate de teneurs respectives $82,67 \pm 0,46 \%$; 82,45 $\pm 0,26 \%$ sont statistiquement identiques. La teneur la plus élevée a été enregistrée au niveau de l'épinard (90,85 $\pm 0,12 \%)$ pendant que la plus faible valeur a été obtenue au niveau des feuilles de manioc $(71,26 \pm 0,25 \%)$.

La valeur énergétique des légumes-feuilles a varié entre 311,75 \pm 0,52 (manioc) et 373,30 \pm 0,43 Cal (épinard). Cette variation a été considérée significative par l'analyse statistique. Par ailleurs, la teneur en fibres varie significativement $(\mathrm{p} \leq 0,05)$ d'un légume-feuille à un autre (Tableau III). Le manioc a présenté la valeur la plus élevée $(10,00 \pm 0,14 \%)$ et l'épinard présente la plus faible $(0,55 \pm 0,07 \%)$.

Tout comme les autres paramètres, l'analyse statistique a montré une différence significative $(\mathrm{p} \leq 0,05)$ au niveau des minéraux (Calcium et Fer). Les feuilles de taro, épinard, patate et manioc de teneurs respectives en calcium 196,38 $\pm 1,30 \mathrm{mg} / 100 \mathrm{~g} ; 111,02 \pm 0,29 \mathrm{mg} / 100 \mathrm{~g} ; 120,39 \pm 0,60$ $\mathrm{mg} / 100 \mathrm{~g}$ et $246,28 \pm 0,67 \mathrm{mg} / 100 \mathrm{~g}$, en Fer 15,07 $\pm 0,24 \mathrm{mg} / 100 \mathrm{~g} ; 19,37 \pm$ $0,65 \mathrm{mg} / 100 \mathrm{~g} ; 27,17 \pm 0,48 \mathrm{mg} / 100 \mathrm{~g}$ et $40,06 \pm 0,07 \mathrm{mg} / 100 \mathrm{~g}$ sont statistiquement différents (Tableau III). Le manioc a présenté la teneur la plus élevée en calcium et en fer pendant que l'épinard et le taro présentent respectivement les teneurs les plus faibles en calcium et en fer.

Tableau III. Paramètres biochimiques des légumes-feuilles

\begin{tabular}{|c|c|c|c|c|c|c|c|}
\hline \multirow[b]{2}{*}{$\begin{array}{l}\text { Légumes } \\
\text {-feuilles }\end{array}$} & \multicolumn{7}{|c|}{ Paramètres biochimiques } \\
\hline & $\begin{array}{c}\text { Lipide } \\
\text { s (\%) }\end{array}$ & $\begin{array}{c}\text { Protéine } \\
\text { s (\%) }\end{array}$ & $\begin{array}{l}\text { Glucide } \\
\text { s (\%) }\end{array}$ & $\begin{array}{l}\text { Fibre } \\
\text { s (\%) }\end{array}$ & $\begin{array}{c}\text { Calciu } \\
\text { m } \\
(\mathrm{mg} / 100 \\
\mathrm{g})\end{array}$ & $\begin{array}{c}\text { Fer } \\
\text { (mg/10 } \\
\text { 0 g) }\end{array}$ & $\begin{array}{l}\text { V E } \\
\text { (cal) }\end{array}$ \\
\hline Taro & $\begin{array}{c}0,62 \pm \\
0,04^{c}\end{array}$ & $\begin{array}{c}3,35 \pm \\
0,13^{\mathrm{a}}\end{array}$ & $\begin{array}{c}82,67 \pm \\
0,46^{\mathrm{a}}\end{array}$ & $\begin{array}{c}6,50 \pm \\
0,00^{\mathrm{a}}\end{array}$ & $\begin{array}{c}196,38 \pm \\
1,30^{\mathrm{a}}\end{array}$ & $\begin{array}{c}15,07 \pm \\
0,24^{\mathrm{a}}\end{array}$ & $\begin{array}{c}349,6 \\
9 \pm \\
2,65^{\mathrm{a}}\end{array}$ \\
\hline Epinard & $\begin{array}{c}0,29 \pm \\
0,02^{b}\end{array}$ & $\begin{array}{c}1,82 \pm \\
0,12^{\mathrm{b}}\end{array}$ & $\begin{array}{c}90,85 \pm \\
0,12^{c}\end{array}$ & $\begin{array}{c}0,55 \pm \\
0,07^{\mathrm{b}}\end{array}$ & $\begin{array}{c}111,02 \pm \\
0,29^{\mathrm{b}}\end{array}$ & $\begin{array}{c}19,37 \pm \\
0,65^{\mathrm{b}}\end{array}$ & $\begin{array}{c}373,30 \\
\pm \\
0,43^{b}\end{array}$ \\
\hline Patate & $\begin{array}{c}0,46 \pm \\
0,01^{\mathrm{a}}\end{array}$ & $\begin{array}{c}2,73 \pm \\
0,11^{\mathrm{c}}\end{array}$ & $\begin{array}{c}82,45 \pm \\
0,26^{\mathrm{a}}\end{array}$ & $\begin{array}{c}4,00 \pm \\
0,14^{c}\end{array}$ & $\begin{array}{c}120,39 \pm \\
0,60^{\mathrm{c}}\end{array}$ & $\begin{array}{c}27,17 \pm \\
0,48^{\mathrm{c}}\end{array}$ & $\begin{array}{c}344,8 \\
4 \pm \\
0,92^{\mathrm{c}}\end{array}$ \\
\hline Manioc & $\begin{array}{c}0,69 \pm \\
0,04^{\mathrm{a}}\end{array}$ & $5,22 \pm 0,18$ & $\begin{array}{c}71,16 \pm \\
0,25^{b}\end{array}$ & $\begin{array}{c}10,00 \pm \\
0,14^{\mathrm{d}}\end{array}$ & $\begin{array}{c}246,28 \pm \\
0,67^{\mathrm{d}}\end{array}$ & $40,06 \pm 0,07$ & $\begin{array}{c}311,75 \\
\pm \\
0,52^{\mathrm{d}}\end{array}$ \\
\hline
\end{tabular}

Les valeurs avec les lettres alphabétiques dans la même colonne sont statistiquement identiques au seuil de $5 \%$ selon le test HSD de Tukey 


\subsection{Paramètres antioxydants des légumes-feuilles}

Les feuilles de taro, épinard, patate et manioc n'ont pas les mêmes taux de vitamine $\mathrm{C}$ (Tableau IV). Le taro et la patate avec des teneurs respectives de $22,92 \pm 0,72 \mathrm{mg} / 100$ g et $24,58 \pm 0,72 \mathrm{mg} / 100 \mathrm{~g}$ sont statistiquement identiques. En outre, le manioc a présenté une teneur élevée en vitamine $\mathrm{C}$ $(40,83 \pm 0,72 \mathrm{mg} / 100 \mathrm{~g})$ et le taro, la plus faible teneur $(22,92 \pm 0,72 \mathrm{mg} / 100$ g) (Tableau IV).

De même que la teneur en vitamine $\mathrm{C}$, l'analyse statistique a montré une différence significative $(\mathrm{p} \leq 0,05)$ au niveau de la teneur en polyphénols des légumes-feuilles analysés (Tableau IV). Le manioc a présenté la teneur la plus élevée (195,34 \pm 0,96 mg/100 g) pendant que l'épinard, la valeur la plus faible $(107,28 \pm 0,74 \mathrm{mg} / 100 \mathrm{~g})$.

Au niveau de la teneur en flavonoïdes des légumes-feuilles, des teneurs de $5,66 \pm 0,24 \mathrm{mg} / 100 \mathrm{~g} ; 2,38 \pm 0,32 \mathrm{mg} / 100 \mathrm{~g} ; 5,89 \pm 0,05 \mathrm{mg} / 100 \mathrm{~g}$ et $7,73 \pm$ $0,20 \mathrm{mg} / 100 \mathrm{~g}$ ont été obtenues respectivement pour le taro, l'épinard, la patate et le manioc. L'analyse statistique a révélé une différence significative $(\mathrm{p} \leq$ $0,05)$ entre ces teneurs. Ainsi, le manioc présente la teneur la plus élevée $(7,73$ $\pm 0,20 \mathrm{mg} / 100 \mathrm{~g})$. Les feuilles de taro $(5,66 \pm 0,24 \mathrm{mg} / 100 \mathrm{~g})$ et de patate $(5,89$ $\pm 0,05 \mathrm{mg} / 100 \mathrm{~g})$ ont des teneurs statistiquement identiques pendant que l'épinard a la valeur la plus faible (2,38 $\pm 0,32 \mathrm{mg} / 100 \mathrm{~g})$.

Tableau IV : Paramètres antioxydants des légumes-feuilles.

\section{Paramètres antioxydants}

\section{Légumes-feuilles}

\begin{tabular}{cccc} 
& Vitamines C & Polyphénols & Flavonoïdes \\
\hline Taro & $22,92 \pm 0,72^{\mathrm{a}}$ & $150,64 \pm 0,71^{\mathrm{a}}$ & $5,66 \pm 0,24^{\mathrm{a}}$ \\
& & & \\
\hline Epinard & $24,58 \pm 0,72^{\mathrm{a}}$ & $107,28 \pm 0,74^{\mathrm{b}}$ & $2,38 \pm 0,32^{\mathrm{b}}$ \\
& & & \\
\hline Patate & $30,00 \pm 1,25^{\mathrm{b}}$ & $167,59 \pm 1,11^{\mathrm{c}}$ & $5,89 \pm 0,05^{\mathrm{a}}$ \\
\hline Manioc & $40,83 \pm 0,72^{\mathrm{c}}$ & $195,34 \pm 0,96^{\mathrm{d}}$ & $7,73 \pm 0,20^{\mathrm{c}}$
\end{tabular}

Les valeurs avec les lettres alphabétiques dans la même colonne sont statistiquement identiques au seuil de $5 \%$ selon le test HSD de Tukey

\subsection{Paramètres antinutritionnels des légumes-feuilles}

L'analyse statistique a montré une différence significative $(\mathrm{p} \leq 0,05)$ au niveau de la teneur en oxalate. Les légumes-feuilles (taro, épinard, patate et manioc) de teneurs respectives $64,75 \pm 0,52 \mathrm{mg} / 100 \mathrm{~g} ; 44,20 \pm 1,27 \mathrm{mg} / 100$ $\mathrm{g} ; 51,38 \pm 0,85 \mathrm{mg} / 100 \mathrm{~g}$ et $64,39 \pm 0,85 \mathrm{mg} / 100 \mathrm{~g}$ sont statistiquement différents (Tableau V). Néanmoins, les légumes-feuilles (taro et manioc) de teneurs respectives $64,75 \pm 0,52$ et $64,39 \pm 0,85$ sont statistiquement 
identiques. Le taro a présenté la teneur en oxalate la plus élevée $(64,75 \pm 0,52$ $\mathrm{mg} / 100 \mathrm{~g})$ et l'épinard, la plus faible (44,20 $\pm 1,27 \mathrm{mg} / 100 \mathrm{~g})$.

Concernant la teneur en phytates, des différences significatives ont été révélées entre les légumes-feuilles étudiés. Les teneurs pour le taro, l'épinard, la patate et le manioc sont respectivement $61,57 \pm 0,89 \mathrm{mg} / 100 \mathrm{~g} ; 65,75 \pm$ $0,65 \mathrm{mg} / 100 \mathrm{~g} ; 48,65 \pm 0,87 \mathrm{mg} / 100 \mathrm{~g}$ et $53,87 \pm 0,43 \mathrm{mg} / 100 \mathrm{~g}$ (Tableau V) sont statistiquement différents selon le test HSD de Tukey (Tableau V). L'épinard a enregistré la teneur la plus élevée $(65,75 \pm 0,65 \mathrm{mg} / 100 \mathrm{~g})$ à l'opposé la patate $(48,65 \pm 0,87 \mathrm{mg} / 100 \mathrm{~g})$ la teneur la plus faible.

Tout comme les teneurs en oxalates et les phytates, les rapports oxalates/calcium et phytates/fer varient significativement $(p \leq 0,05)$ d'un légume-feuille à un autre (Tableau $\mathrm{V})$. Le rapport oxalates/calcium a oscillé entre $0,26 \pm 0,00$ et ; $0,43 \pm 0,01$ pendant que celui du phytates/fer a oscillé entre 1,44 $\pm 0,01$ et 4,08 $\pm 0,12$. En outre, les rapports les plus élevés ont été enregistrés au niveau des feuilles de patate $(0,43 \pm 0,01)$ et de taro $(4,08 \pm$ 0,12 ) respectivement pour oxalates/calcium et phytates/fer. Les feuilles de manioc ont présenté les valeurs les plus faible pour les rapports oxalates/calcium $(0,26 \pm 0,00)$ et phytates/fer $(1,44 \pm 0,01)$.

\section{Paramètres antinutritionnels}

\begin{tabular}{ccccc}
$\begin{array}{c}\text { Légumes- } \\
\text { feuilles }\end{array}$ & $\begin{array}{c}\text { Oxalates } \\
(\mathbf{m g} / \mathbf{1 0 0} \mathbf{~ g})\end{array}$ & $\begin{array}{c}\text { Phytates } \\
(\mathbf{m g} / \mathbf{1 0 0} \mathbf{g})\end{array}$ & Oxalates / Calcium Phytates / Fer \\
\hline Taro & $64,75 \pm 0,52^{\mathrm{a}}$ & $\begin{array}{c}61,57 \pm \\
0.89^{\mathrm{a}}\end{array}$ & $0,33 \pm 0,00^{\mathrm{a}}$ & $4,08 \pm 0,12^{\mathrm{a}}$ \\
& & & & \\
& & $65,75 \pm$ & & \\
Epinard & $44,20 \pm 1,27^{\mathrm{b}}$ & $0.65^{\mathrm{b}}$ & $0,4 \pm 0,01^{\mathrm{b}}$ & $3,40 \pm 0,09^{\mathrm{b}}$ \\
& & & & \\
& & $48,65 \pm$ & & \\
Patate & $51,38 \pm 0,85^{\mathrm{c}}$ & $0.87^{\mathrm{c}}$ & $0,43 \pm 0,01^{\mathrm{c}}$ & $1,79 \pm 0,01^{\mathrm{c}}$ \\
& & & & \\
& & $53,87 \pm$ & & \\
Manioc & $64,39 \pm 0,85^{\mathrm{a}}$ & $0.43^{\mathrm{d}}$ & $0,26 \pm 0,00^{\mathrm{d}}$ & $1,44 \pm 0,01^{\mathrm{d}}$ \\
\hline
\end{tabular}

Tableau V. Paramètres antinutritionnels des légumes-feuilles

Les valeurs avec les lettres alphabétiques dans la même colonne sont statistiquement identiques au seuil de $5 \%$ selon le test HSD de Tukey

\section{Discussion}

En Afrique, diverses plantes sont connues tant pour leurs propriétés médicinales qu'alimentaires. Les résultats de nos enquêtes ont montré que 100 $\%$ des familles connaissent les légumes-feuilles. La diversité et la connaissance des légumes-feuilles sont liées à la demande des 
consommateurs, et, en particulier, à la grande diversité des communautés culturelles (Dongmo et al., 2005).

La cueillette et le maraichage sont les deux principales sources d'approvisionnement en légumes-feuilles, avec une prédominance pour le maraîchage. Plus de 64 \% de familles enquêtées dans cette localité obtiennent les légumes-feuilles par culture. A Lomé (Togo) aussi, Kanda et al. (2009) ont observé que la source principale de légumes-feuilles est le maraîchage. Selon ces auteurs, ce type d'approvisionnement permet de maintenir la diversité des légumes-feuilles.

La fréquence de consommation moyenne des légumes-feuilles par mois par individu, dans cette zone d'étude, est de trois à quatre fois. Cette fréquence de consommation est inférieure à la fréquence de consommation observée par Konan et al. (2015) qui ont montré que la fréquence de consommation des légumes-feuilles par mois par individu à Abidjan et à Bouaké était de cinq fois contre 10 fois à Korhogo.

D'autres facteurs pouvant influencer la consommation des légumesfeuilles sont leur disponibilité, leur goût et la facilité de cuisson (Dansi et al., 2008). Toutes les familles enquêtées consomment ces légumes-feuilles préparés à l'état cuit sous forme de sauce et pâte. Au bénin par contre, une étude de Agbankpé et al. (2014) a montré que les légumes-feuilles sont préparés à l'état frais ou séchés (poudres) puis consommés comme légume d'accompagnement (sauce) ou utilisés comme tisane.

Certains légumes-feuilles sauvages possèdent des propriétés médicinales et ne sont consommées que pour soigner diverses maladies telles que le paludisme, les parasites intestinaux, les infections (Chweya et Eyzaguire, 1999). Les résultats de nos enquêtes ont montré que 70 \% de la population utilisent les légumes-feuilles à des fins médicinales.

L'analyse physico-chimique des différents légumes-feuilles a révélé une grande variabilité pour la plupart des paramètres étudiés. En ce qui concerne le $\mathrm{pH}$, il détermine l'acidité. Ses valeurs obtenues varient de 6,22 \pm 0,02 à $6,67 \pm 0,01$. Ces résultats sont similaires à ceux trouvés par Suthep et al. (2016) dans la laitue (légume à feuilles vertes) $(6,64 \pm 0,78$ à 6,89 $\pm 0,98)$.

$\mathrm{Au}$ niveau du taux d'humidité, l'analyse statistique montre que les teneurs des légumes-feuilles varient entre 78,02 $\pm 0,25$ et 93,75 $\pm 0,12 \%$. Dorosz (1999) a trouvé des valeurs qui variaient entre 70 à $90 \%$ dans les légumes-feuilles. Ces valeurs correspondent à celles trouvées dans la présente étude. Le taux d'humidité élevé de ces produits indique que les quatre variétés de feuilles sont des denrées périssables.

Les cendres représentent environ 1 à $5 \%$ de la masse d'un aliment sur une base humide. Les résultats de la présente étude $(0,79 \pm 0,01$ à $0,95 \pm 0.01$ \%) sont inférieurs à ceux de Prisacaru et al. (2017) qui ont trouvé comme teneur en cendre 2,82 \% lors de l'estimation des niveaux de métaux lourds 
dans les légumes à feuilles vertes. Ils sont également inférieurs à ceux de Gwarzo (2012) qui a trouvé une teneur en cendre de 4,72 \pm 0,27 \% au Nigéria lors d'une étude sur la détermination de la teneur en iode de certaines substances couramment utilisées comme légumes-feuilles.

Les différents légumes-feuilles ont un degré Brix variant de 2,16 \pm 0,47 à 7,00 $\pm 0,00{ }^{\circ}$ Brix. Ces valeurs sont inférieures à celle du Codex Alimentarius (2005) (20 à $55^{\circ} \mathrm{Brix}$ ) pour les légumes servant de sauce.

Par ailleurs, les légumes-feuilles de la ville de Daloa sont très pauvres en lipides $(0,29 \pm 0,02$ à $0,69 \pm 0,04 \%)$. Ces valeurs sont inférieures à celles rapportées par Moussa et al. (2007) dans les légumes-feuilles (0,2 à 1 mg/100 g).

Les protéines sont des nutriments pouvant renouveler les cellules et sont nécessaires à la croissance des enfants. Les teneurs en protéine comprises entre 1,82 $\pm 0,12$ et 5,22 $\pm 0,18 \%$ sont inférieures à celles obtenues par Moussa et al. (2007) (Manihot esculenta Crantz $=7 \%$; Moringa oleifera L. = 8,1 \%) et Mbemba et al. (2013) (Cuervea integrifolia A. = 9,56 \%).

Les teneurs en glucides des légumes-feuilles sont comprises entre $71,16 \pm 0,25$ et $90,85 \pm 0,12 \%$. Ces glucides sont principalement concentrés sous forme d'amidon (FAO, 1997). Les feuilles riches en glucides et protéines, constituent des sources d'énergie.

Les minéraux sont des composants importants du régime alimentaire humain, car ils servent de cofacteurs à de nombreux processus physiologiques et métaboliques. Les analyses des paramètres ont montré que les teneurs en minéraux diffèrent les unes des autres dans cette étude. Le fer est nécessaire à la production d'hémoglobine et au transport de l'oxygène des poumons au travers des vaisseaux sanguins (Nelson \& Cox, 2005). Les teneurs en fer des légumes-feuilles analysés comprises entre 15,07 $\pm 0,24$ et 40,06 $\pm 0,07 \mathrm{mg} / 100$ g sont similaires à celles trouvées dans les légumes-feuilles (09 à 56 mg/100 g de MS) par Anin et al. (2012). Les légumes-feuilles constituent une bonne source de fer qui pourrait aider au recouvrement d'un problème nutritionnel comme l'anémie et d'autres déficiences en micronutriments (Nangula et al., 2010). Par ailleurs, le calcium a un effet sur la santé des os en termes de protection (Park \& Weaver, 2012). Il est un facteur majeur

dans l'ossification, joue un rôle dans la contraction musculaire, et l'absorption de la vitamine B12 (Mensah et al., 2008). Les teneurs en calcium comprises entre 111,02 $\pm 0,29$ et 246,28 $\pm 0,67 \mathrm{mg} / 100 \mathrm{~g}$ sont inférieures à celles trouvées dans les légumes-feuilles par Odhav et al. (2007) (2067 à 2363 $\mathrm{mg} / 100 \mathrm{~g}$ ). Les légumes-feuilles de la ville de Daloa ont des teneurs faibles en calcium. Cela pourrait être lié à l'origine diverse (champs, autres sites maraîchers et jardins) des légumes-feuilles (Agbo et al., 2009). 
Les légumes-feuilles renferment des fibres $(0,55 \pm 0,07$ à $10,00 \pm 0,14$ $\%)$. Il est admis que les fibres interviennent notablement dans le transit intestinal. La consommation des légumes-feuilles présente donc un intérêt.

Les valeurs énergétiques des différents légumes-feuilles comprises entre 311,75 $\pm 0,52$ et 373,30 $\pm 0,43$ Cal sont inférieures à celles de Moussa et al. (2007) (57 à 110,2 kcal).

Dans les légumes-feuilles analysés, il a été noté la présence des polyphénols, des flavonoïdes, de vitamines $\mathrm{C}$, des oxalates, des phytates. Les cinq groupes chimiques identifiés jouent des rôles importants dans l'organisme tant humain qu'animal et permettent d'expliquer certaines propriétés curatives que les tradipraticiens prêtent aux feuilles des légumes. Les teneurs en polyphénols des feuilles de taro, épinard, patate et manioc sont respectivement de 150,64 \pm 0,71 mg/100 g ; 107,28 \pm 0,74 mg/100 g ; 167,59 $\pm 1,11 \mathrm{mg} / 100 \mathrm{~g}$ et $195,34 \pm 0,96 \mathrm{mg} / 100 \mathrm{~g}$. La teneur en polyphénols des feuilles analysés est supérieure à celle trouvée par Jabbari et al. (2007) dans l'épinard (60,69 à 98,82 mg/100 g). Ces polyphénols constituent une famille de molécules organiques largement présente dans le règne végétal. Ils prennent une importance croissante, notamment grâce à leurs effets bénéfiques sur la santé (Stanley et al., 2003). En effet, leur rôle d'antioxydants naturels suscite de plus en plus d'intérêt pour la prévention et le traitement du cancer (Chen et al., 2004), des maladies inflammatoires (Laughton et al., 1991), cardiovasculaires (Frankel et al., 1993) et neurodégénératives (Orgogozo et al., 1997).

Quant aux flavonoïdes, ils sont des diphénols possédant des propriétés vitaminiques et ayant une action antioxydante en captant les radicaux libres engendrés par exemple par l'inflammation, l'hypoxie et les irradiations (Harper et al., 1982). Leur teneur dans les légumes-feuilles varie significativement d'un type à un autre. Dans la présente étude, le manioc a enregistré la teneur la plus élevée $(7,73 \pm 0,20 \mathrm{mg} / 100 \mathrm{~g})$ et l'épinard a présenté la plus faible $(2,38 \pm 0,32 \mathrm{mg} / 100 \mathrm{~g})$. Selon Harbone \& Williams (2000), les flavonoïdes ont des activités antivirales, antibactériennes, antifongiques, anti-inflammatoires, antiallergiques et antitumorales.

Les teneurs en vitamines $C$ de nos légumes-feuilles sont de 22,92 \pm 0,72 à 40,83 $\pm 0,72 \mathrm{mg} / 100 \mathrm{~g}$. Ces valeurs se rapprochent de celles trouvées par Soro et al. (2012) (15,55 à 42,22 mg/100 g). Les richesses des légumesfeuilles en vitamines $C$ correspondent à des enjeux de santé particulièrement significatifs dans les pays où l'on constate de nombreux cas d'anémie causés par le paludisme, et de déficience immunitaire.

A l'exception de la patate $(51,38 \pm 0,85 \mathrm{mg} / 100 \mathrm{~g}$ ) (même teneur en oxalate que la morelle), les valeurs en acide oxalique des feuilles de taro, épinard et manioc analysés $(64,75 \pm 0,52 \mathrm{mg} / 100 \mathrm{~g} ; 44,20 \pm 1,27 \mathrm{mg} / 100 \mathrm{~g}$; et $64,39 \pm 0,85 \mathrm{mg} / 100 \mathrm{~g}$ ) sont supérieures à celles de Sheela et al. (2004) 
(célosie $=24,33 \mathrm{mg} / 100 \mathrm{~g}$, morelle $=50,62 \mathrm{mg} / 100 \mathrm{~g}$ ). Sachant que l'acide oxalique est un antagoniste de l'utilisation du calcium, le rapport oxalate/calcium a été déterminé dans la présente étude. Dans les légumesfeuilles analysés, le rapport oxalates/calcium $(0,26 \pm 0,00$ à $0,43 \pm 0,01)$ est inférieur à 2,25 (Tchiegang \& Kitikil, 2004). Ce faible taux permet de limiter le risque d'absorption (Betsche \& Fretzdorff, 2005).

Les teneurs en phytates que présentent les différentes feuilles analysées sont inférieures à celles de Elke \& Zannini (2013) dans le sarrasin (Fagopyrum esculentum Moench) (1000 mg/100 g). Par ailleurs, la biodisponibilité du fer peut être estimée par son rapport molaire avec les phytates. En effet, des études ont montré que l'acide phytique est le principal agent chélateur réduisant la biodisponibilité des cations divalents (Weaver \& Kannan, 2002). Les différents légumes-feuilles de taro, épinard, patate et manioc ont un ratio variant de $1,44 \pm 0,01$ à $4,08 \pm 0,12$. Ces valeurs sont inférieures à celles déterminées par Saha et al. (1994) dans les régimes à base de farine de blé (10 à 14). La présence donc des phytates, chélateurs des minéraux divalents diminuent leur biodisponibilité.

\section{Conclusion}

L’objectif du présent travail était de caractériser les légumes-feuilles les plus consommés dans le centre-ouest de la Côte d'Ivoire (Daloa). Les résultats des enquêtes ont montré que cette localité renferme une multitude de légumes-feuilles. Les plus consommés de ces légumes-feuilles ont été : le taro, l'épinard, la patate et le manioc. Quatre (04) groupes de paramètres ont été évalués dans ces feuilles. Il s'agit des paramètres physico-chimiques $(\mathrm{pH}$, acidité, humidité et degré Brix), des composés biochimiques (fibres, lipides, protéines, glucides totaux, valeur énergétique, fer et calcium), des composés antioxydants (vitamine $\mathrm{C}$, flavonoïde et polyphénols totaux) et des composés antinutritionnels (oxalates et phytates). L'exemple des quatre (4) espèces analysées montre l'importance des légumes dans l'alimentation humaine. La proportion des fibres justifie leur importance dans le transit intestinal. Dans ces légumes, la présence des antioxydants est capable de contrer l'action néfaste des oxydants comme les radicaux libres. Ces légumes-feuilles constituent également une source de Fer pour l'organisme. Pour les nombreux effets bénéfiques, il importe d'intégrer certains légumes-feuilles sauvages ou mi-sauvages dans des vrais systèmes agraires.

\section{References:}

1. AFNOR (1986). Recueil de normes françaises. Contrôle de la qualité des produits laitiers. Afnor, Paris-la-Défense.

2. AFNOR (1984). Essais des eaux - Détermination de la concentration totale en calcium et 
3. magnésium - Méthode titrimétrique à l'EDTA (NF T90-003).

4. AFNOR (1974). Produits dérivés des fruits et légumes - Détermination de l'acidité titrable. NF V05-101 ; 4 P.

5. Agbankpé A. J., Dougnon T. V., Bankole H. S., Yèhouénou B., Yedomonhan H., $\quad$ Legonou M. \& Dougnon T. J. (2014). Etude ethnobotanique des légumes feuilles thérapeutiques utilisés dans le traitement des diarrhées au sud-Bénin (Afrique de l'Ouest). International Journal of Biological and Chemical Sciences,8(4) : 17841795

6. Agbo E., Kouamé C., Mahyao A., N’Zi J. C. \& Fondio L. (2009). Nutritional importance of Indigenous Leafy Vegetables in Côte d'Ivoire. Acta Hort. 806, ISHS, 1 : 361-366.

7. Almekinders C. \& Boef W. (2000). Encouraging diversity. The conservation and development of plant genetic resources. Intermediate Technology. Publication, $\quad$ London (UK), 14 P.

8. Anin L. O. A., Soro L. C., Kouamé C., Agbo E. A. \& Kouadio K. K. A. (2012). Valeur nutritionnelle des légumes-feuilles consommés en Côte d'Ivoire. International Journal of Biological and Chemical Sciences, 6(1) : 128-135

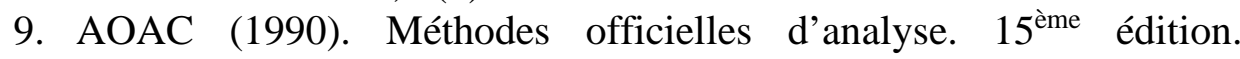
Association des chimistes analytiques officiels, Washington, DC, Etats-Unis, pp. 200-210.

10. Atwater W. \& Rosa E. (1899). A new respiratory colorimeter and the conservation of $9: 214-251$.

energy in human body. Physiological. Reviews,

11. Betsche T. \& Fretzdorff B. (2005). Biodegradation of oxalic acid from spinach using cereal radicles. Journal of Agricultural and Food Chemistry, vol. 53, ${ }^{0} 25$, p. 9751-8

12. Chen D., Kenyon G. D., Kuhn D. J., Kazi A., \& Bhuivan M. (2004). Green tea and tea polyphenols in cancer prevention. Frontiers in Bioscience, vol. 9, $\mathrm{n}^{\circ} 2618$

13. Chweya J. A. \& Eyzaguirre P. (eds.) (1999). The biodiversity of traditional leafy vegetables. IPGRI Rome (Italy), $182 \mathrm{p}$.

14. Codex Alimentarus (2005). Programme mixte FAO/OMS sur les normes alimentaires. Examen en vue de l'élaboration de normes, directives ou autres textes pour les aliments dérivés des biotechnologies. Groupe intergouvernemental spécial du codex sur les aliments dérivés des biotechnologies, 5 ème session, $40 \mathrm{P}$.

15. Dansi A., Adjatin A., Adoukonou-Sagbadja H., Falade V., Yedomonhan H., Odou D. \& Dossou B. (2008). Traditional leafy vegetables and their use in the Benin Republic. Genetic Resources and Crop Evolution, 55: 1239-1256. 
16. Day R.A. \& Underwood A.L. (1986). Quantitative analysis. In: Prentice-Hall. $701 \mathrm{P}$.

17. Dongmo T., Gockowski J., Hernandez S. \& Mbang R. (2005). L'agriculture périurbaine à Yaoundé : ses rapports avec la réduction de la pauvreté, le développement économique, la conservation de la biodiversité et de l'environnement. Tropicultura 23, 130-135.

18. Dorosz P. (1999). Vitamins, sels minéraux, oligo-éléments Ed Maloine. Paris. 101P.

19. Elke K. A. \& Zannini E. (2013). Cereal Grains for the Food and Beverage Industries . 512p

20. FAO (1997). Vitamin and mineral requirements in human nutrition. Report of a joint Expert consultation, 1ème edition, 341p

21. FAO (1978). La situation mondiale de l'alimentation et de l'agriculture. Collection Agriculture $\mathrm{N}^{\circ}$ 9, 199 P.

22. Frankel F. R., Allemand J. B., Kinsella J. E. \& Kanner J. (1993). Inhibition of oxidation of human low-density lipoprotein by phenolic substances in red wine. Lancet, vol. 341, $\mathrm{n}^{\circ}$ 454-457.

23. Gwarzo U. S. (2012). Determination of iodine content of some commonly utilized leafy vegetables: spinacea oleracea linn (spinach), brassica oleracea var (cabbage), hibiscussabdr1ffa linn and lactuca sativa l. (lettuce) found in kano metropolis vegetable markets. Chemsearch Journal 3(2): 11 - 13.

24. Harborne J. B. \& Williams C. (2000). Advances in flavonoid research since 1992. Phytochemistry, 55 (6) : 481-504.

25. Harper H. A., Rodwel V. W. \& Mayes P. A. (1982). Précis de biochimie. Presses. Universitaires de Laval, Québec, Canada. 138p

26. Jabbari A., Barzegar M., Erfani F. \& Hassandokht M-R., (2007). Effect of Cultivar on Chemical Composition of Some Iranian Spinach. Pakistan Journal of Biological Sciences, 10 : 602-606.

27. Kanda M., Wala K., Batawila K., Djaneye-Boundjou G., Ahanchede A., Akpagana K., (2009). Le maraîchage périurbain à Lomé : pratiques culturales, risques sanitaires et dynamiques spatiales. Cahiers Agricultures, 18(4) : 356-363.

28. Konan Y., Mamidou W. K., Kagoyire K. (2015). Contribution des Légumes Feuilles à la Nutrition des Populations en Zones Urbaines de la Côte D'ivoire. European Journal of Scientific Research, 130 (4) : 338-351.

29. Latta M. \& Eskin M. (1980). A simple method for phytate determination. Journal of Agricultural and Food Chimistry, 28. 1313-1315pp.

30. Laughton M. J., Evans P. J., Moroney M. A., Hoult J. R., Halliwell B. (1991). Inhibition of mammalian 5-lipoxygenase and cyclo-oxygenase 
by flavonoids and phenolic dietary additives. Relationship to antioxidant activity and to iron ion-reducing ability. Biochemical Pharmacology, 42 (9) : 1673-1681.

31. Mbemba F., Moutsambote J. M., Nzikou J. M., Mvoula-Tsiery M., Itoua Okouango S. Y., $\quad$ Nganga I., Mboungou Z. \& Silou T. (2013). Factures Nutritional Value of the traditional Picking Vegetable, cuervea isangiensis (De wild.) N. hallé in CongoBrazzaville. Advance Journal of Food Science and Technology 5(1) : 72-76.

32. Meda A., Lamien C. E., Romito M., Millogo J. \& Nacoulma O. G. (2005). Determination of the total Phenolic, Flavonoid and Proline contents in Burkina Fasan Honey, as well as their radical scavenging activity. Food Chemistry, 91(3) : 571-577.

33. Mensah J. K., Okoli R. I., Ohaju-Obodo J. O. \& Eifediyi K. (2008). Phytochemical, nutritional and medical properties of some leafy vegetables consumed by Edo people of Nigeria. African Journal of Biotechnology, 7(14) : 2304-2309.

34. Mnzava, N. A. (1997). Comparing nutritional values of exotic and indigenous vegetables. In R. Schippers and L. Budd eds., African Indigenous vegetables, ODA, UK, pp. 70-75

35. Moussa N., Wade S., Dossou N., Amadou T. \& Rokhaya D. (2007). Valeur nutritionnelle du Moringa oléifera, étude de la biodisponibilité du fer, effet d'enrichissement de divers plats traditionnels Sénégalais avec la poudre des feuilles. African journal of Food Agriculture Nutrition and Development, vol. 7, no.3, 2007.

36. Nangula P. U., Oelofse A., Kwaku G. D., Megan J. B. \& Mieke F. (2010). Nutritional value of leafy vegetables of sub-Saharan Africa and their potential contribution to human health: A review. Journal of Food Composition and Analysis, 23 : 499-509.

37. Nelson D. L. \& Cox M. M. (2005). Lehninger Principles of Biochemistry. Biochemistry and Molecular Biology Education, 33(1) : 74-75.

38. Odhav B., Beekrum S., Akula U. \& Baijnath H. (2007). Preliminary assessment of nutritional value of traditional leafy vegetables in KwaZulu-Natal, South Africa. Journal of Food Composition and Analysis, 20 : 430-435.

39. Orgogozo J. M., Dartigues J. F., Lafont S., Letenneur L. \& Commenges D. (1997). Wine consumption and dementia in the elderly: A prospective community study int he Bordeaux area. Rev. Neurol. vol. 153, p. 185-192 
40. Park C. Y \& Weaver C. M. (2012). Review Vitamin D Interactions with Soy Isoflavones on Bone after Menopause : A Review. Nutrients., 4 : 1610-1621.

41. Pongracz G., Weiser H. \& Matzinger D. (1971). Tocopherols Antioxydant. Fat. Science Technology, (97), 90-104.

42. Prisacaru A. E., Apostol L. C. \& Ropciuc S. (2017). Estimation of heavy metal levels in green leafy vegetables purchased from suceava. Journal of Faculty of Food Engineering, Stefan cel Mare University of Suceava, Romania Volume XVI, pp $234-238$.

43. Rubaihayo E. B. (1996). Contrition des légumes indigènes à la sécurité alimentaire des ménages. African Crop Science Journal, African Crop Science Conference Proceedings (3), 1337-1340.

44. Saha P. R., Weaver C. M. \& Mason A. C. (1994). Mineral bioavailability in rats from intrinsically labeled whole wheat flour of various phytate levels. Journal of agricultural and food chemistry, $42: 2531-5$.

45. Sheela K., Kamal N. G., Vijayalakshmi D., Geeta M. \& Roopa B. P. (2004). Proximate Composition of Underutilized Green Leafy Vegetables in Southern Karnataka. Journal of Human Ecology, 15 (3) : 227-229.

46. Singleton V. L., Orthofer R. \& Lamuela-Raventos R. M. (1999). Analysis of total phenols and other oxidant substrates and antioxidants by means of Folin-Ciocalteu reagent. Methods in Enzymology, 299, 152-178.

47. Stanley M. A. Beck J. G. \& Swann A. C. (2003). Antioxidants and the free radical theory of degenerative Disease. Journal consulting and clinic psychology, 71(2). 309p.

48. Suthep S., Prat I., Pisit V. \& Kraichat T. (2016). Uptake of Copper and Zinc in Lettuce (vegetable to green leaf) Planted in Sida Soil and Lignite Bottom Ash Mixtures. Naresuan University Journal: Science and Technology ; (25)3. Pp. 31-42

49. Tchiegang C. \& Kitikil A. (2004). Données ethnonutritionnelles et caractéristiques physico- chimiques des légumes feuilles consommés dans la savane de l'Adamaoua (Cameroun).

Tropicultura 22 (1), 11-18.

50. Vaughan J. P. \& Morrow R.H. (1991). Manueld'epidemiologie pour la gestion de la santé au niveau du district. Programme special PNUD/Banque mondiale/OMS de recherche et de formation concernant les maladies tropicales, P.195.

51. Vodouhe S., Dovoedo A., Anihouvi V. B., Tossou R. C. \& Soumanou M. M. (2012). 
52. Influence du mode de cuisson sur la valeur nutritionnelle de Solanum macrocarpum, Amaranthus hybridus et Ocimum gratissimum, trois légumes-feuilles traditionnels acclimatés au Bénin. International Journal of Biological and Chemical Sciences, 6(5): 1926-1937.

53. Weaver C. M. \& Kannan S. T. (2002). Phytate and mineral bioavailability. In: Reddy NR, Sathe SK, eds. Food phytates. Boca Raton, FL: CRC Press : 211-224. 